\title{
Actinomyces as a Cause of Unexplained Fever Diagnosed by Next-Generation Sequencing: Case Report and Review
}

Xiu Huang ( $\sim$ huangxiu17@mails.jlu.edu.cn )

People's Hospital of Shanxi Province https://orcid.org/0000-0002-1368-4080

$\mathrm{Na} \mathrm{Du}$

The First Hospital of Jilin University

jiaxue He

The First Hospital of Jilin University

yujun Du

The First Hospital of Jilin University

\section{Case report}

Keywords: fever of unknown origin, endobronchial actinomycosis, next-generation sequencing

Posted Date: January 7th, 2021

DOI: https://doi.org/10.21203/rs.3.rs-139943/v1

License: (a) (i) This work is licensed under a Creative Commons Attribution 4.0 International License. Read Full License 


\section{Abstract}

Background: Actinomycosis is a rare cause of unexplained origin fever (FUO). The NGS, particularly in cases of FUO in conventional microbiology, is a promising and advantageous technology for earlier diagnosis and more success. It gives new insights in the management of FUO, especially in infectious diseases.

Case presentation: We present a case of a 71-year-old woman who had FUO that the etiology was endobronchial actinomycosis eventually diagnosed by next-generation sequencing of bronchoalveolar lavage fluid. She was admitted to the our department with intermittent fever up to $40.0^{\circ} \mathrm{C}$, chills, cough with white sputum, chest distress and slightly dyspnea over one month. According to special clinical course and laboratory data of the patient, she was given intravenous sulbenicillin sodium for 11 days and oral amoxicillin at $500 \mathrm{mg}$ every $8 \mathrm{~h}$ for another 1 month. When she returned for follow-up, she reported a notable improvement in her cough, dyspnea, sputum production and fever that was completely absent.

Conclusion: In obscure causes of FUO, clinicians should consider the possibility of thoracic actinomycosis and actively use next-generation sequencing to help diagnose.

\section{Background囚}

Fever of unknown origin (FUO) is identified with fevers of $38.3^{\circ} \mathrm{C}$ or greater for 3 or more weeks that is not still diagnosed after a systemic inpatient/outpatient examination for 3 days. The most common causes of unexplained origin fever are infectious diseases. Actinomycosis is a rare chronic invasive bacterial infection caused by Actinomyces that is always difficult to be separated. In the last few years, there have been some better methods for detecting Actinomyces. For example, the next-generation sequencing (NGS) is a promising and rising technique to help to diagnose Actinomycosis. And with the next-generation sequencing technologies being increasingly less expensive, it should be made full use of diagnosing infectious disease processes in the future. Here, we present a case of a 71-year-old woman who had FUO that the etiology was endobronchial actinomycosis eventually diagnosed by nextgeneration sequencing of bronchoalveolar lavage fluid.

\section{Case Presentation】}

A 71-year-old non-smoking married old woman was admitted to the first hospital of Jilin university in May 2019 , after suffering from intermittent fever up to $40.0^{\circ} \mathrm{C}$, chills, cough with white sputum, chest distress and slightly dyspnea over one month. The patient's history indicated no chest pain, hemoptysis, night sweats, nausea, vomiting, diarrhea, abdominal pain or special travel history. Weight loss was about $7 \mathrm{~kg}$ in the last 20 days. She had medical history of eczema and hypertension for many years, but no pasthistory of respiratory system disease. Unfortunately, she suffered a car accident which disabled left upper limb 20 years ago. On admission, her vital signs were: body temperature(axilla) $38.7^{\circ} \mathrm{C}$, pulse rate 110 beats/min, respiratory rate 20 breaths/min, blood pressure $135 / 77 \mathrm{mmHg}$. Laboratory investigations 
showed neutrophilic granulocyte percentage was 0.83 , elevated level of C-reactive protein was $145.00 \mathrm{mg} / \mathrm{L}$ and hemocyte sedimentation rate was $75 \mathrm{~mm} / 1 \mathrm{~h}$. The total leukocyte, renal function, procalcitonin, creatine kinase and tumor markers including carcinoembryonic antigen and neuron specific enolase were normal and HIV, HBV and HCV were negative. Besides, purified protein derivative (PPD) was not reactive. Tuberculosis T cell spot method (serum) was also negative. Interestingly, lymphocytes was $0.79 \times 10^{\wedge} 9$ (range of normal value:1.10-3.20). Cardiac ultrasound showed a slightly widened pulmonary artery, reduced left ventricular diastolic function, calcified aortic valve, and atherosclerotic plaque in the ascending aorta. The computed tomography (CT) images of the chest revealed parts of the bronchus were not clear with atelectasis, the bronchial wall was slightly thickened and there was some pleural effusion in the middle lobe of the right lung (Fig a). And there were many pulmonary lesions that tended to be inflammatory with diameters of 0.2 to $0.3 \mathrm{~cm}$. In order to definite the cause of the thickening of the bronchial wall and the nature of the lesions, electronical bronchoscope and bronchoalveolar lavage were performed. Since the patient complained of dyspnea duration the operation, mucosal biopsy and brush examination were not conducted. Bronchoscopy revealed the bronchial openings in the inner basal segment of the lower lobe of the left lung were occluded and the supply of blood was slightly rich in the smooth mucosa (Fig b). The bacterial culture of bronchoalveolar lavage fluid (BALF) did not recover any pathogen, cytology suggested macrophages and lymphocytes and acid-fast smear was negative. Because of recurrent fever and the particularity of her bronchial lesion, we performed molecular identification by next-generation sequencing from the BALF. The report listed all microorganisms with valid data that could be detected in the sample (Fig c): veillonella parvula (138), veillonella atypica (10), streptococcus parasanguinis (89), actinomyces (60), actinomyces gerencseriae (4), prevotella melaninogenica (49), prevotella salivae (19), rothia dentocariosa (54), rothia mucilaginosa (3) and candida albicans (8). Over the one month preceding her admission, the patient presented an equivocal response. She was prescribed cephalosporin before entered our hospital for about 10 days. Once admitted by our hospital later, according to her symptoms, laboratory and imaging findings, community acquired pneumonia was suspected and she was treated with moxifloxacin for 13 days. With the course of treatment, her temperature tended to be intermittent fever, cough and dyspnea even the antibiotic regimen treatment was on-going. At that time, NGS results were reported. According to her special clinical course and laboratory data, she was given intravenous sulbenicillin sodium for 11 days and oral amoxicillin at $500 \mathrm{mg}$ every $8 \mathrm{~h}$ for another 1 month. When she returned for follow-up, she reported a notable improvement in her cough, dyspnea, sputum production and fever that was completely absent.

\section{Discussion And Conclusions:}

Despite advanced diagnostic methods and therapeutic approach, the differential diagnosis of FUO remains to be one of the sophisticated challenges all over the world. The most common causes of FUO can be classified into four main etiology: infections, malignancy, non-infectious inflammatory disorders (NIID, such as autoimmune and rheumatic diseases, vasculitis syndromes and granulomatous disorders), and miscellaneous conditions[1]. However, infections caused by various $b$ pathogens are still the leading cause for classical FUO around the world especially in developing countries [1, 2]. 
Actinomyces are a genus of gram-positive, rod-shaped, non-acid-fast, non-spore forming anaerobic or microaerophilic bacteria with a tendency to produce branching filaments and are the normal colonizing flora of the oral cavity, pharynx, gastrointestinal and genitourinary tract[3]. Six of these are considered to be pathogenic in humans, including $A$. israelii, A. naeslundi, $A$. odontolyticus, $A$. viscosus, A. meyeri and $A$. gerencseriae[4]. They can affect many sites of humans, such as the cervicofacial (50-60\%), thoracic actinomycosis $(15-20 \%)$, abdominopelvic $(20 \%)$, the central nervous system (2\%), and rarely involves cutaneous, ophthalmic, musculoskeletal disease, pericarditis, genitourinary or disseminated disease[5]. As for thoracic actinomycosis, the most common etiology of infection is poor dental hygiene and diabetes, HIV infection, malignancies, steroid, obesity, alcoholism, and age $>65$ years are risk factors for developing thoracic actinomycosis[6]. Otherwise, patients with chronic obstructive pulmonary disease, pulmonary sequelae following tuberculosis and with the aspiration of foreign bodies[7, 8], are considered to also be risk factors[9].

Dyspnea, cough, expectoration, hemoptysis and chest pain are the most common and typical presenting symptoms[10]. In some developing countries, blood-stained sputum is also main one symptom[11]. Interestingly, recurrent high fever was the most primary symptom the patient had in our report.

To separate, culture, and ultimately diagnosis of thoracic actinomycosis are always challenging and delayed. There are some reasons as follows. Radiologic modalities are usually inadequate for diagnosing and only could provide some useful information such as chronic segmental airspace consolidation that irregularly have thickened bronchial walls with central necrotic low attenuation areas and peripheral enhancement, a mass-like shadow, cavitation, pleural thickening, pleural effusions, hilar or mediastinal lymphadenopathy, bronchial and bronchiolar dilatation[12-15]. However, the typical CT feature of thoracic actinomycosis is only seen in less than $50 \%$ of patients as a retrospective study reported[16]. The best standard of thoracic actinomycosis was that actinomyces colonies was visualized in histology. However, the rate of Actinomyces isolation using culture tests is only approximately $7 \%$ and a retrospective, multicenter study reported that up to $40 \%$ of cases were culture negtive[17]. In our case, culture tests of the BALF were negative result. Using conventional microbiological tools of several attempts of samples, we could not diagnose what kind of infection in our patient. Fortunately, with the development of techniques, the Bruker MALDI-TOF BioTyper MS (MBT) and 16S rRNA gene sequencing have emerged as new reliable tools for the identification of Actinomyces[18, 19] in recent years. And some recent studies have shown that next-generation sequence (NGS) may significantly enhance the rare or unusual microbiological diagnostic of the culture-negative specimens in several clinical situations by allowing rapid identification of bacteria in different samples, such as blood samples, sputum, body fluids, or tissues [20-22]. Subsequently, we performed molecular identification by NGS from the BALF sample and finally detected the microbe. Meantime, there were also other bacteria and fungi in this specimen by NGS, indicating that contamination from the oral cavity was a consideration.

We think that the real pathogen of our patient isn't other microbes that just are contamination than $A$. gerencseriae because of the following reasons: first the patient was treated with cephalosporin and moxifloxacin to cover common gram-positive and negative bacillias, however, they are not effective. 
Secondly, there are 6 organisms that are members of oral microflora, constituting the flora of the patient's mouth identified by next-generation sequencing in the patient. $V$. parvula has been implicated in septicemia, meningitis, endocarditis, peritonitis and osteomyelitis [23, 24] , however, it is generally considered to be contaminant bacteria. V.atypica is one early-colonizing members of the dental plaque biofilm[25], but to our knowledge have not been recognized as causative agents of disease in respiratory system. There are only occasional reports of endocarditis due to $S$. parasanguinis[26]. P. melaninogenica is often associated with pyomyositis[27]. Interestingly, $P$. salivae can be isolated from the mouths of humans, but has not been reported as a pathogen in humans. Moreover, $R$. dentocariosa and $R$. mucilaginosa is usually recognized as an opportunistic pathogen with keratitis, endocarditis, peritonitis, arthritis and bacteremia[28-32]. Of course, R. mucilaginosa is reported with pneumonia, it usually affects immunocompromised hosts as the review reports[33]. Candida albicans infect humans only under certain conditions such as immunodeficiency. In contrast, the ability of $A$. gerencseriae to act as infectious agents is known. However, it's difficult to isolate this pathogen from the involved samples by normal ways because the specimen must be collected under strictly anaerobic conditions, and mixed infections are inevitable. Finally, we decided to do an experimental treatment for $A$. gerencseriae diagnosed by NGS and the clinical recovery of our patient also indicated that the infection was caused by $A$. gerencseriae.

DNA sequencing technologies were invented in the early 1970's and the NGS revolution appeared due to cut down the cost and time of sequencing in 2005[34]. They are useful in a variety of areas such as cancer, haematology, neurology and epidemiology[35-37]. Because it takes less time and less priori knowledge of the type of microbial to come to an conclusion, there are many promising applications on epidemiology including: infectious disease diagnostics, hospital infection microbial surveillance programs, community outbreak investigations and antimicrobial resistance[38]. However, there are challenges for NGS to widely apply to clinical treatment. For example, detecting many microorganisms directly from a patient sample requires physicians to determine that which is the real pathogen and which is likely (but not absolutely) a contaminant for a patient with non-specific symptoms. Morever, it takes more money than other conventional tools. With the development of the NGS, there is no doubt that it will become more and more useful and reliable to help solve medical diagnostic dilemmas.

Although thoracic actinomycosis is relatively difficult to isolate and identify, prognosis is relatively good if it is properly treated. There are no definitive and standard guidelines for kinds of drugs and duration of antibiotics treatment for thoracic actinomycosis yet, penicillin $\mathrm{G}$ has been recommended as the preferred drug of choice for treatment. The optimal treatment duration is 2 to 6 weeks of intravenous penicillin followed oral penicillin or amoxicillin for 6 to 12 months $[39,40]$. When the patients are allergic to penicillin, tetracyclines are available for mild presentations. Worth mentioned is that actinomyces are anaerobic, metronidazole should be avoided in the treatment of pulmonary actinomycosis[41]. Moreover, surgical intervention might be necessary if we don't evaluate the nature of the lesions and alleviate severe symptoms[42]. In our case, this old woman was treated with intravenous sulbenicillin for 11 days and further treated with oral amoxicillin for 1 month. 
To the best of our knowledge, this case is meaningful and instructive because it's the first case of NGS is used for thoracic actinomycosis, that is a cause of FUO. The NGS, particularly in cases of FUO in conventional microbiology, is a promising and advantageous technology for earlier diagnosis and more success. It gives new insights in the management of FUO, especially in infectious diseases.

In obscure causes of FUO, clinicians should consider the possibility of thoracic actinomycosis and actively use next-generation sequencing to help diagnose.

\section{Declarations}

Ethics approval and consent to participate: Yes.

Consent for publication: Yes.

Availability of data and materials: Yes.

Competing interests: Not applicable.

Funding: Not applicable.

Authors' contributions: Miss Huang contributed to the completion of the first draft. Mrs. Du contributed to the main ideas and treatment. Mrs. He contributed to the data and figure about results. Yujun Du Ph.D. was responsible for the review and final completion of the paper.

Acknowledgements: I would like to thank Director Wanguo Bao for the most accurate diagnosis and treatment.

Actinomyces as a cause of unexplained fever diagnosed by next-generation sequencing: case report and review

\section{References}

1. Arnow PM, Flaherty JP. Fever of unknown origin. Lancet. 1997;350(9077):575-80. doi:10.1016/s0140-6736(97)07061-x.

2. Mulders-Manders $C$, Simon A, Bleeker-Rovers C. Fever of unknown origin. Clinical medicine (London, England). 2015;15(3):280-4. doi:10.7861/clinmedicine.15-3-280.

3. Li J, Li Y, Zhou Y, Wang C, Wu B, Wan J. Actinomyces and Alimentary Tract Diseases: A Review of Its Biological Functions and Pathology. BioMed research international. 2018;2018:3820215. doi:10.1155/2018/3820215.

4. Mabeza GF, Macfarlane J. Pulmonary actinomycosis. The European respiratory journal. 2003;21(3):545-51. doi:10.1183/09031936.03.00089103. 
5. Kononen E, Wade WG. Actinomyces and related organisms in human infections. Clin Microbiol Rev. 2015;28(2):419-42. doi:10.1128/cmr.00100-14.

6. Paulo CO, Jordao S, Correia-Pinto J, Ferreira F, Neves I. Actinomycosis, a lurking threat: a report of 11 cases and literature review. Rev Soc Bras Med Trop. 2018;51(1):7-13. doi:10.1590/0037-8682-02152017.

7. Kassab K, Karnib M, Bou-Khalil PK, Bizri AR. Pulmonary actinomycosis presenting as postobstructive pneumonia. International journal of infectious diseases: IJID : official publication of the International Society for Infectious Diseases. 2016;48:29-31. doi:10.1016/j.ijid.2016.04.009.

8. Shin JH, Hong YH, Kim KS. Actinomycosis associated with a retained pencil core for a period of 13 years. Emergency medicine Australasia: EMA. 2015;27(2):176-7. doi:10.1111/1742-6723.12356.

9. Valour F, Senechal A, Dupieux C, Karsenty J, Lustig S, Breton P, et al. Actinomycosis: etiology, clinical features, diagnosis, treatment, and management. Infection drug resistance. 2014;7:183-97. doi:10.2147/idr.S39601.

10. Kim SR, Jung LY, Oh IJ, Kim YC, Shin KC, Lee MK, et al. Pulmonary actinomycosis during the first decade of 21st century: cases of 94 patients. BMC Infect Dis. 2013;13:216. doi:10.1186/1471-233413-216.

11. Zhang M, Zhang XY, Chen YB. Primary pulmonary actinomycosis: a retrospective analysis of 145 cases in mainland China. The international journal of tuberculosis lung disease: the official journal of the International Union against Tuberculosis Lung Disease. 2017;21(7):825-31. doi:10.5588/ijtld.16.0773.

12. Kim TS, Han J, Koh WJ, Choi JC, Chung MJ, Lee JH, et al. Thoracic actinomycosis: CT features with histopathologic correlation. AJR American journal of roentgenology. 2006;186(1):225-31. doi:10.2214/ajr.04.1749.

13. Mato N, Oshikawa K, Sakuma Y, Sawai T, Ohno S, Sugiyama Y. [Thoracic actinomycosis: clinical, radiological, and pathological findings in 11 cases]. Nihon Kokyuki Gakkai zasshi $=$ the journal of the Japanese Respiratory Society. 2003;41(8):514-20.

14. Heo SH, Shin SS, Kim JW, Lim HS, Seon HJ, Jung SI, et al. Imaging of actinomycosis in various organs: a comprehensive review. Radiographics: a review publication of the Radiological Society of North America Inc. 2014;34(1):19-33. doi:10.1148/rg.341135077.

15. Morland D, Hassler S. Case 219: Pelvic Actinomycosis Mimicking Malignant Tumor. Radiology. 2015;276(1):304-8. doi:10.1148/radiol.2015131224.

16. Sun XF, Wang P, Liu HR, Shi JH. A Retrospective Study of Pulmonary Actinomycosis in a Single Institution in China. Chin Med J. 2015;128(12):1607-10. doi:10.4103/0366-6999.158316.

17. Bonnefond S, Catroux M, Melenotte C, Karkowski L, Rolland L, Trouillier S, et al. Clinical features of actinomycosis: A retrospective, multicenter study of 28 cases of miscellaneous presentations. Medicine. 2016;95(24):e3923. doi:10.1097/md.0000000000003923.

18. Fong P, Francis MJ, Hamblin JF, Korman TM, Graham M. Identification and diversity of Actinomyces species in a clinical microbiology laboratory in the MALDI-TOF MS era. Anaerobe. 2018;54:151-8. 
doi:10.1016/j.anaerobe.2018.09.007.

19. Yamamoto S, Takegawa H, Taniike N, Takenobu T. Actinomycotic Osteomyelitis of the Mandible Diagnosed Using Matrix Assisted Laser Desorption Ionization-Time of Flight Mass Spectrometry: A Case Report. Journal of oral maxillofacial surgery: official journal of the American Association of Oral Maxillofacial Surgeons. 2018;76(10):2122-30. doi:10.1016/j.joms.2018.04.020.

20. Salipante SJ, Hoogestraat DR, Abbott AN, SenGupta DJ, Cummings LA, Butler-Wu SM, et al. Coinfection of Fusobacterium nucleatum and Actinomyces israelii in mastoiditis diagnosed by nextgeneration DNA sequencing. J Clin Microbiol. 2014;52(5):1789-92. doi:10.1128/jcm.03133-13.

21. Gu W, Miller S, Chiu CY. Clinical Metagenomic Next-Generation Sequencing for Pathogen Detection. Annual review of pathology. 2019;14:319-38. doi:10.1146/annurev-pathmechdis-012418-012751.

22. Leo S, Gaia N, Ruppe E, Emonet S, Girard M, Lazarevic V, et al. Detection of Bacterial Pathogens from Broncho-Alveolar Lavage by Next-Generation Sequencing. Int J Mol Sci. 2017;18(9). doi:10.3390/ijms18092011.

23. Borchardt KA, Baker M, Gelber R. Veillonella parvula septicemia and osteomyelitis. Ann Intern Med. 1977;86(1):63-4. doi:10.7326/0003-4819-86-1-63.

24. Li J, Chen P, Li J, Gao X, Chen X, Chen J. A new treatment of sepsis caused by veillonella parvula: A case report and literature review. J Clin Pharm Ther. 2017;42(5):649-52. doi:10.1111/jcpt.12559.

25. Johnson BP, Jensen BJ, Ransom EM, Heinemann KA, Vannatta KM, Egland KA, et al. Interspecies signaling between Veillonella atypica and Streptococcus gordonii requires the transcription factor CcpA. J Bacteriol. 2009;191(17):5563-5. doi:10.1128/jb.01226-08.

26. Hashmi AT, Khalid M, Waseem H, Batool A, Patel J, Kamholz S. Necrotizing Crescentic Glomerulonephritis Complicating Bivalvular Bacterial Endocarditis. Cureus. 2018;10(4):e2520. doi:10.7759/cureus.2520.

27. Odeh M, Oliven A, Potasman I, Solomon H, Srugo I. Pyomyositis of the thigh due to Prevotella melaninogenica. Infection. 2000;28(1):49-50. doi:10.1007/s150100050012.

28. Williams B, Jafri M, Arfeen S, Reidy JJ, Hariprasad SM, Farooq AV. Atypical Keratitis Caused by Rothia Dentocariosa. Ocul Immunol Inflamm. 2019:1-3. doi:10.1080/09273948.2019.1659829.

29. Boudewijns M, Magerman K, Verhaegen J, Debrock G, Peetermans WE, Donkersloot P, et al. Rothia dentocariosa, endocarditis and mycotic aneurysms: case report and review of the literature. Clinical microbiology and infection: the official publication of the European Society of Clinical Microbiology and Infectious Diseases. 2003;9(3):222-9. doi:10.1046/j.1469-0691.2003.00503.x.

30. Keng TC, Ng KP, Tan LP, Chong YB, Wong CM, Lim SK. Rothia dentocariosa repeat and relapsing peritoneal dialysis-related peritonitis: a case report and literature review. Ren Fail. 2012;34(6):804-6. doi:10.3109/0886022x.2012.678208.

31. Kaasch AJ, Saxler G, Seifert H. Septic arthritis due to Rothia mucilaginosa. Infection. 2011;39(1):812. doi:10.1007/s15010-010-0065-5.

32. Yeung DF, Parsa A, Wong JC, Chatur N, Salh B. A case of Rothia dentocariosa bacteremia in a patient receiving infliximab for ulcerative colitis. Am J Gastroenterol. 2014;109(2):297-8. 
doi:10.1038/ajg.2013.366.

33. Maraki S, Papadakis IS. Rothia mucilaginosa pneumonia: a literature review. Infectious diseases (London, England). 2015;47(3):125-9. doi:10.3109/00365548.2014.980843.

34. Margulies M, Egholm M, Altman WE, Attiya S, Bader JS, Bemben LA, et al. Genome sequencing in microfabricated high-density picolitre reactors. Nature. 2005;437(7057):376-80. doi:10.1038/nature03959.

35. van Dijk EL, Auger $H$, Jaszczyszyn $Y$, Thermes $C$. Ten years of next-generation sequencing technology. Trends Genet. 2014;30(9):418-26. doi:10.1016/j.tig.2014.07.001.

36. Moorcraft SY, Gonzalez D, Walker BA. Understanding next generation sequencing in oncology: A guide for oncologists. Crit Rev Oncol Hematol. 2015;96(3):463-74. doi:10.1016/j.critrevonc.2015.06.007.

37. Kuo FC. Next generation sequencing in hematolymphoid neoplasia. Semin Hematol. 2019;56(1):2-6. doi:10.1053/j.seminhematol.2018.05.006.

38. Goldberg B, Sichtig H, Geyer C, Ledeboer N, Weinstock GM. Making the Leap from Research Laboratory to Clinic: Challenges and Opportunities for Next-Generation Sequencing in Infectious Disease Diagnostics. mBio. 2015;6(6):e01888-15. doi:10.1128/mBio.01888-15.

39. Choi J, Koh WJ, Kim TS, Lee KS, Han J, Kim H, et al. Optimal duration of IV and oral antibiotics in the treatment of thoracic actinomycosis. Chest. 2005;128(4):2211-7. doi:10.1378/chest.128.4.2211.

40. Wong VK, Turmezei TD, Weston VC. Actinomycosis. BMJ. 2011;343:d6099. doi:10.1136/bmj.d6099.

41. Steininger $C$, Willinger $B$. Resistance patterns in clinical isolates of pathogenic Actinomyces species. J Antimicrob Chemother. 2016;71(2):422-7. doi:10.1093/jac/dkv347.

42. Park JY, Lee T, Lee H, Lim HJ, Lee J, Park JS, et al. Multivariate analysis of prognostic factors in patients with pulmonary actinomycosis. BMC Infect Dis. 2014;14:10. doi:10.1186/1471-2334-14-10.

\section{Figures}



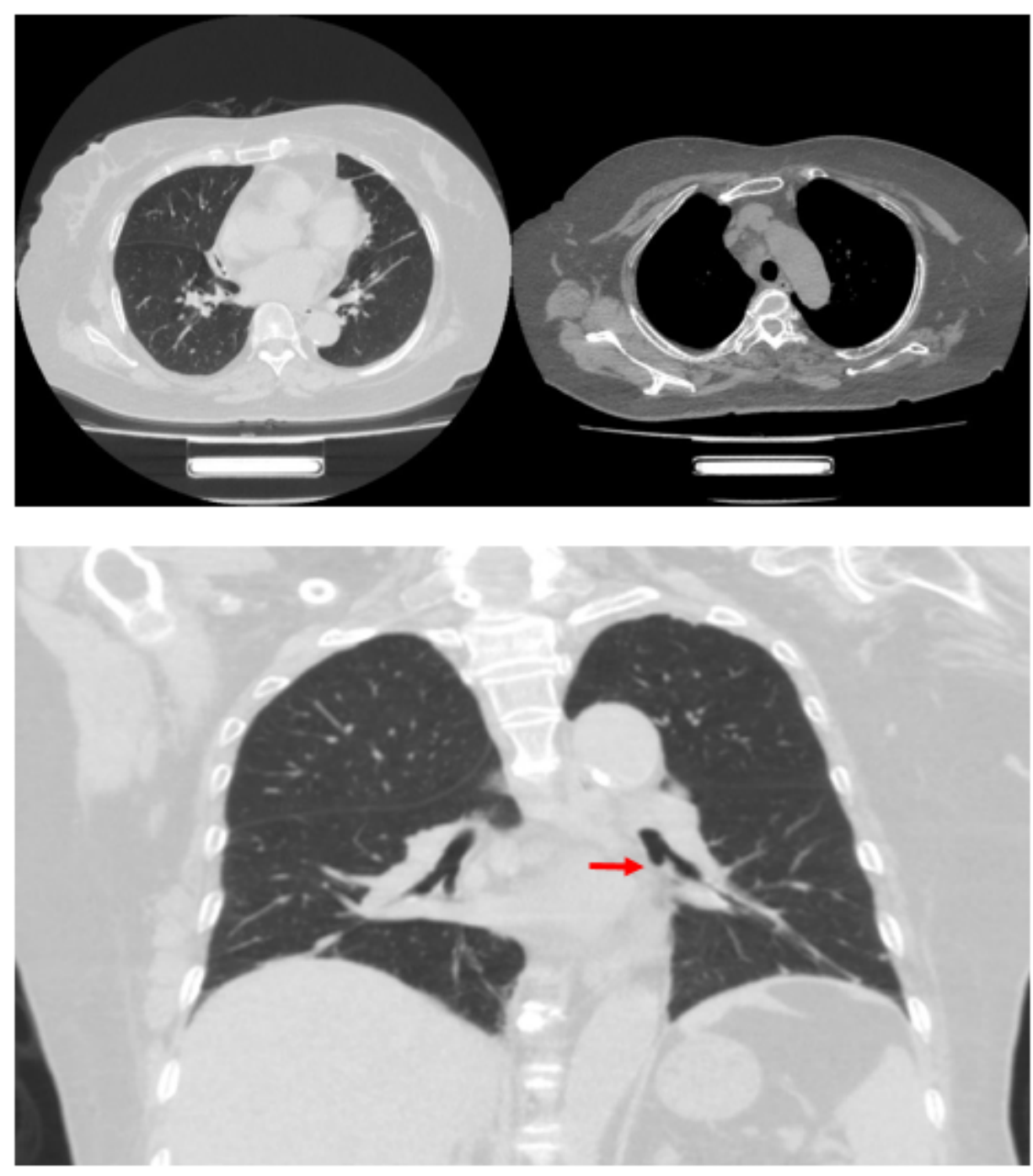

\section{Figure 1}

The computed tomography (CT) images of the chest revealed the bronchial wall was slightly thickened and there was some pleural effusion in the middle lobe of the right lung 


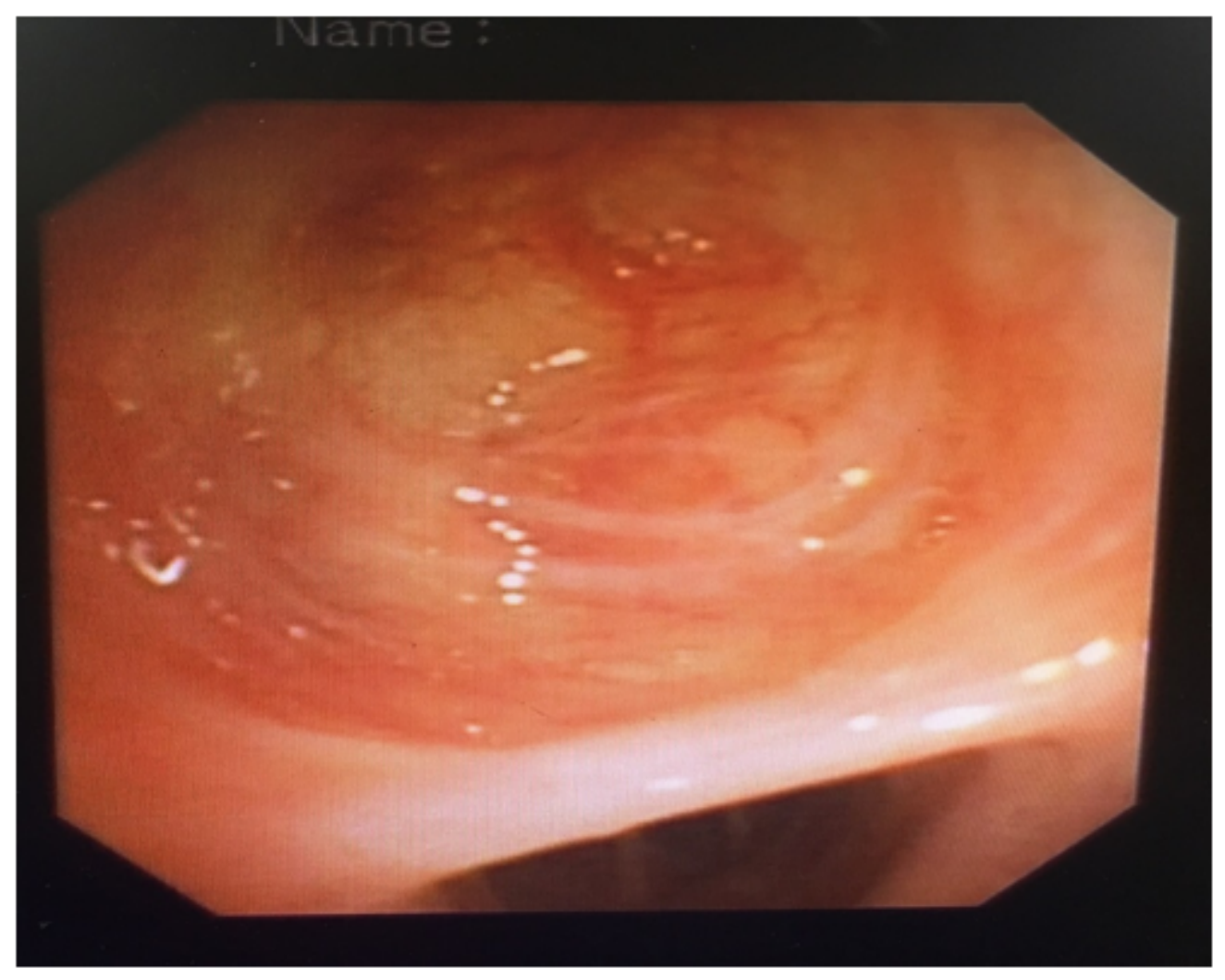

Figure 2

Bronchoscopy demonstrates the bronchial openings in the inner basal segment of the lower lobe of the left lung were completely occluded

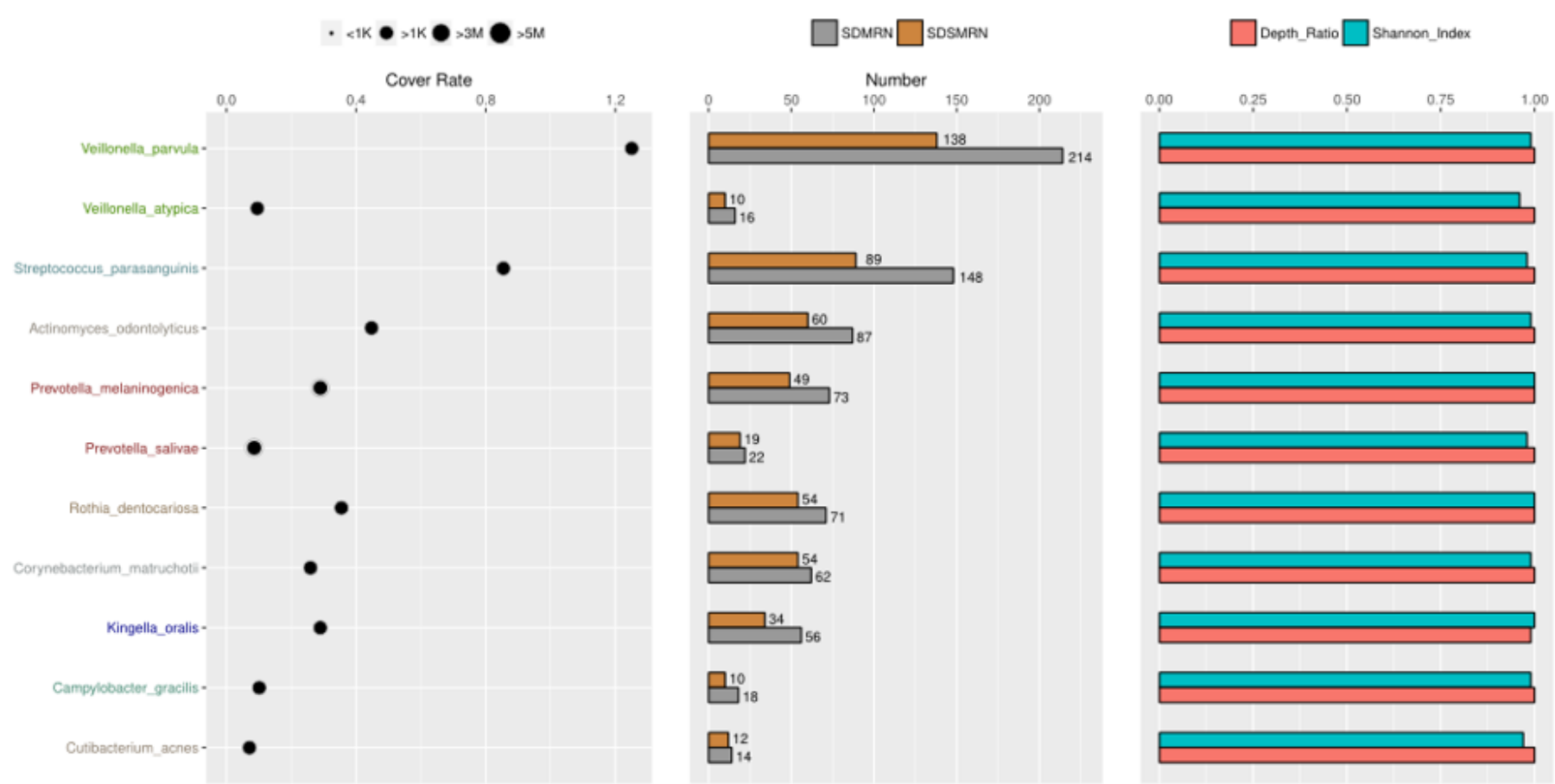

Figure 3 
all microorganisms with valid data in BALF 